\title{
Use of Glucagon-Like-Peptide 1 Receptor Agonists and Risk of Fracture as Compared to Use of Other Anti-hyperglycemic Drugs
}

\author{
Johanna H. M. Driessen ${ }^{1,2,3}$ - Hein A. W. van Onzenoort ${ }^{3,4} \cdot$ Jakob Starup-Linde $^{5,6}$ • \\ Ronald Henry $^{7,8}$ - Andrea M. Burden ${ }^{1,2,3}$ - Cees Neef ${ }^{1,2} \cdot J_{0 o p}$ P. van den Bergh ${ }^{9,10}$. \\ Peter Vestergaard ${ }^{5,11} \cdot$ Frank de Vries $^{1,2,3,12}$
}

Received: 18 May 2015/Accepted: 4 July 2015/Published online: 17 July 2015

(C) The Author(s) 2015. This article is published with open access at Springerlink.com

\begin{abstract}
Glucagon-like peptide-1 receptor agonists (GLP-1 RAs) are a new class of drugs that might have a potential beneficial effect on bone metabolism. Data on the effect of GLP-1 RAs and fracture risk are lacking. The aim of the present study was to investigate the association between the use of GLP-1 and the risk of fracture. A casecontrol study was performed using Danish National Health Service data. Cases were those who sustained a fracture and controls were those without a fracture during the study period (2007-2011), all aged 18 years and above. Conditional logistic regression estimated the odds ratios (OR) of fracture with current use of DPP4-I use. Analyses were adjusted for comorbidities and recent drug use. Among cases ( $n=229,114$ ), there were 6993 current non-insulin anti-diabetic drug (NIAD) users (excluding incretin users) and 255 GLP-1 RA users. Similarly, among controls ( $n=229,114), 7209$ were NIAD users (excluding incretin users) and 220 were GLP-1 RA users. Current GLP-1 RA use was not associated with a decreased risk of fracture
\end{abstract}

Frank de Vries

f.devries@uu.nl

1 Division of Pharmacoepidemiology and Clinical Pharmacology, Utrecht Institute of Pharmaceutical Sciences, Utrecht University, Utrecht, The Netherlands

2 Care and Public Health Research Institute (CAPHRI), Maastricht, The Netherlands

3 Department of Clinical Pharmacy and Toxicology, Maastricht University Medical Centre+, Maastricht, The Netherlands

4 Department of Pharmacy, Radboud University Nijmegen Medical Centre, Nijmegen, The Netherlands

5 Department of Clinical Medicine, Aalborg University, Aalborg, Denmark [adjusted (adj.) OR 1.16; $95 \%$ CI 0.83-1.63]. Osteoporotic fracture risk was also not associated with current GLP-1 RA use (adj. OR 0.78; 95 \% CI 0.44-1.39). In our nationwide case-control study, we identified that the use of GLP1 RA was not associated with fracture risk as compared to the use of other anti-hyperglycemic drugs. Additionally, current GLP-1 RA use, stratified by cumulative or average daily dose, is not associated with fracture risk. Further research should focus on long-term use of GLP-1 RA and fracture risk.

Keywords GLP-1 RA - Fracture - Type 2 diabetes mellitus · Case-control

6 Department of Endocrinology and Internal Medicine, Aarhus University Hospital, Aarhus, Denmark

7 Department of Medicine, Maastricht University Medical Centre+, Maastricht, The Netherlands

8 Cardiovascular Research Institute Maastricht, Maastricht University Medical Centre+, Maastricht, The Netherlands

9 Department of Internal Medicine, Maastricht University Medical Centre+, Maastricht, The Netherlands

10 Department of Internal Medicine, VieCuri Medical Centre, Venlo, The Netherlands

11 Department of Endocrinology, Aalborg University Hospital, Aalborg, Denmark

12 MRC Epidemiology Lifecourse Unit, Southampton General Hospital, Southampton, UK 


\section{Introduction}

The risk of fracture is significantly increased in patients with type 2 diabetes mellitus (T2DM) [1]. It has been hypothesized that a reduced bone strength or bone quality plays a role in patients with T2DM [2]. Alternatively, it has been suggested that anti-hyperglycemic drugs might affect fracture risk. For instance, observational studies showed that the use of thiazolidinediones is associated with a 1.3-1.9-fold [3, 4] increased risk of fracture, in particular in women, as compared to the use of other anti-hyperglycemic drugs. Insulin use has been associated with an elevated fracture risk [5], whereas metformin might be associated with a reduced fracture risk [6].

Glucagon-like peptide-1 receptor agonists (GLP-1 RAs), such as exenatide and liraglutide, are a new class of drugs in the treatment of T2DM. GLP-1 RAs may have a potential beneficial effect on bone metabolism, established by binding of GLP-1 RAs to a GLP-1 receptor on osteocytes and osteoblasts, as shown in in vitro studies [7-9]. Consequently, this may result in an increased bone formation and a decreased bone resorption $[8,10]$. As a result of this process, we hypothesize that this then may lead to a reduced risk of fracture.

However, currently, there are limited data, particularly epidemiologic, in the literature. A recent meta-analysis of randomized clinical trials $(n=4255$, mean duration of included studies 67.4 weeks) did not show a reduced risk of fracture with the use of GLP-1 RAs [11] as compared to the use of other anti-hyperglycemic drugs. To our knowledge, there is only one observational study examining fracture risk with GLP-1 RA use which showed no association [12]. In particular, studies with longer durations of use or stratified to daily dose are required to best understand the association between GLP-1 RA use and fracture risk. We therefore sought to examine the association between GLP-1 RA use compared to other anti-hyperglycemic drug use and fracture risk in a nation-wide casecontrol study.

\section{Methods}

\section{Data Source}

We utilized data from the Danish National Health Service, which cover all contacts to the health sector, and include approximately 5.2 million individuals in 1995 and 5.5 million in 2011 [13]. The unique 10-digit civil registry number was used to link population-based registries and generate a complete hospital discharge and prescription history for each individual [14]. Data on vital status (e.g., change of address and date of death) for the entire Danish population have been collected since 1968 in the Civil Registration System. All inpatient contacts have been registered through the Danish National Hospital Discharge Register [15] since 1977, and outpatient visits to hospitals, outpatient clinics, and emergency rooms have been included since 1995. In Denmark, prescription records are sent directly to a Register of Medicinal Product Statistics (i.e., a prescription database) at the Danish Medicines Agency. The prescription database includes information on patient's civil registry number, the type and amount of drug prescribed according to the Anatomical Therapeutic Chemical (ATC) classification system, and the date when the prescription was filled. Pharmacy data have been collected since January 1, 1996. This study was subject to control by the National Board of Health and the Danish Data Protection Agency.

\section{Study Design}

The study was designed as a case-control study. Cases were all subjects, both genders and aged 18 years and above, who sustained a fracture, low or high trauma, (International Classification of Diseases and Related Health Problems (ICD)-10 codes: S02, S12, S22, S32, S42, S52, S62, S72, S82, S92, T02, T08, T10, and T12) between 9 May 2007 (the first ever prescription of a GLP-1 RA in Denmark) and 31 December 2011. Controls were all subjects, both genders and aged 18 years and above, who did not sustain a fracture during the study period. We randomly selected one control for each case, matched by gender and year of birth. The controls were selected using incidence density sampling [16]. The date of the first fracture was used as the index date for cases, and controls were assigned the index date of their matched case.

Fractures were classified into the following categories using ICD-10 codes: hip (S72.0-S72.2), radius/ulna (S52), and vertebrae (S12, S22.0-S22.1, S32.0-S32.2, S32.7, $\mathrm{S} 32.8, \mathrm{~T} 08)$. A major osteoporotic fracture was defined as a fracture of the hip, radius/ulna, vertebrae, or humerus (S42.2-S42.4) according to the WHO definition [17].

\section{Exposure of Interest}

We identified all drugs bought during the observation period using the Register of Medicinal Product Statistics. The dose of the drug was expressed as defined daily dose (DDD) [18]. ATC code A10B was used to determine exposure to non-insulin anti-diabetic drugs (NIAD), and patients were classified as current (1-91 days) or past (over 91 days) NIAD users, based on the time of the most recent prescription before the index date. Current NIAD users 
were divided into two mutually exclusive categories: never incretin users and incretin users.

To control for the potential that diabetes might act as a confounder [2], we employed never incretin use (i.e., current NIAD use excluding incretin use) as the reference category in our analysis. ATC codes A10BX04 and A10BX07 were used to determine GLP-1 RA exposure before the index date in the prescription database. Based on the time since the most recent prescription, cases and controls were classified as current (1-91 days), recent (92-182), or past (over 182 days) GLP-1 RA users. The DDDs estimated the cumulative dose of GLP-1 RAs for current GLP-1 RA users, expressed as exenatide equivalents. The average daily dose was estimated by dividing the cumulative exposure by the treatment time (time between the first GLP-1 RA prescription and the index date).

\section{Potential Confounders}

A history of the following potential confounders ever before the index date were taken into account: chronic obstructive pulmonary disease (COPD), previous fracture, rheumatoid arthritis, hypothyroidism, hyperthyroidism, cancer, retinopathy, alcoholism, secondary osteoporosis (diabetes type 1 , hypogonadism or premature menopause), and congestive heart failure. The potential confounders were identified from the National Hospital register using ICD-10 and ICD-8 codes. Additional potential confounders included a prescription in the 6 months before the index date of the following drugs: dipeptidyl peptidase 4 inhibitors (DPP4-Is), oral glucocorticoids [19], lipid-modifying drugs, antidepressants [20], anxiolytics, hypnotics [21], antipsychotics, anti-Parkinson drugs [22], antihypertensives [beta-blockers, thiazide diuretics, renin-angiotensinaldosterone system (RAAS) inhibitors, calcium channel blockers, and loop diuretics], and antiarrhythmics. The prescription database was used to explore the presence of a prescription of the above-mentioned drugs.

\section{Statistical Analysis}

Conditional logistic regression estimated the association between the use of GLP-1 RAs versus use of other antihyperglycemic drugs and risk of fracture, using the SAS 9.3 software. Analyses were stratified by age, gender, type of fracture, and for current GLP-1 RA use also by average daily dose and cumulative exposure. The stratified analyses were determined a priori, therefore no interaction analyses was performed. As a sensitivity analysis, we adjusted for current metformin use, as it has been associated with a decreased risk of fracture [6]. As a second sensitivity analyses, we adjusted the final model for insulin use, as insulin use has been associated with an increased fracture risk [5]. Final regression models were determined by stepwise backward elimination using a significance level of 0.05 . All results are presented as odds ratios (OR) with the corresponding $95 \%$ confidence intervals (CI).

\section{Results}

\section{Study Population}

The study population consisted of 229,145 cases and the same number of controls. The mean age was 55 years, and $56 \%$ were women. Baseline characteristics are shown in Table 1.

Among cases, we identified 6993 (3.1\%) current NIAD users (excluding incretin users) and 255 (0.1\%) GLP-1 RA users (current, recent, or past). Similarly, we identified 7209 (3.1\%) NIAD users (excluding incretin users), and $220(0.1 \%)$ GLP-1 RA users among controls. The mean duration of actual GLP-1 RA use (from first GLP-1 RA prescription till index date) was 36 weeks.

\section{GLP-1 RA Use and Risk of Any Fracture}

Current GLP-1 RA was not associated with a decrease in fracture risk: adjusted (adj.) OR 1.16, $95 \%$ CI 0.83-1.63. Similarly, no significant decrease was observed for recent use (adj. OR 1.03, $95 \%$ CI 0.69-1.53), or for past use (adj. OR 1.09, $95 \%$ CI 0.82-1.46). Having no prior use of NIAD (never use) was associated with a significant increase in risk of fracture (adj. OR 1.10, $95 \%$ CI 1.06-1.14), as well as past NIAD use (adj. OR 1.12, $95 \%$ CI 1.05-1.20). The risk of fracture was not reduced after stratification by sex or age (Table 2).

\section{Other Fracture Types}

Table 3 shows that current GLP-1 RA use was not associated with osteoporotic fracture risk (adj. OR 0.78, $95 \%$ CI 0.44-1.39). We identified similar trends for recent GLP1 RA use (adj. OR 0.97, $95 \%$ CI 0.52-1.79) and for past use (adj. OR 0.94, $95 \%$ CI 0.60-1.48). Stratification by sex and age did not substantially change the results (data not shown). There was no significant association with fracture risk of the radius/ulna for current GLP-1 RA use (adj. OR 0.93, $95 \%$ CI 0.48-1.80), recent GLP-1 RA use (adj. OR 1.32, $95 \%$ CI 0.55-3.17), or for past GLP-1 RA use (adj. OR 0.93, $95 \%$ CI 0.48-1.80). Stratification by gender and age did not show a decreased fracture risk (data not shown). Hip and vertebral fracture risk was not associated with GLP-1 RA use (current, recent, or past), Table 3. 
Table 1 Baseline characteristics

\begin{tabular}{|c|c|c|}
\hline Characteristic & Cases $(n=229,145)$ & Controls $(n=229,145)$ \\
\hline Women & $127,449(55.6)$ & $127,449(55.6)$ \\
\hline Mean age at index date (years, SD) & $55(20.6)$ & $55(20.6)$ \\
\hline $18-49$ years & $90,598(39.5)$ & $90,607(39.5)$ \\
\hline $50-59$ years & $37,247(16.3)$ & $37,191(16.2)$ \\
\hline $60-69$ years & $38,751(16.9)$ & 38,805 (16.9) \\
\hline 70-79 years & $28,950(12.6)$ & 28,931 (12.6) \\
\hline $80+$ years & $33,599(14.7)$ & $33,611(14.7)$ \\
\hline \multicolumn{3}{|l|}{ History of comorbidities } \\
\hline Type 1 diabetes & $2113(0.9)$ & $1419(0.6)$ \\
\hline Alcoholism & $11,147(4.9)$ & $4824(2.1)$ \\
\hline Fracture & $46,446(20.2)$ & $15,418(6.7)$ \\
\hline Hyperthyroidism & $3715(1.6)$ & $3688(1.6)$ \\
\hline Hypothyroidism & $2887(1.3)$ & $2496(1.1)$ \\
\hline COPD & $10,812(4.7)$ & $7,418(3.2)$ \\
\hline Congestive heart failure & $7141(3.1)$ & $5424(2.4)$ \\
\hline Cancer & $21,893(9.6)$ & $18,486(8.1)$ \\
\hline Rheumatoid arthritis & $3912(1.7)$ & $2795(1.2)$ \\
\hline Retinopathy & $3105(1.4)$ & $2314(1.0)$ \\
\hline Neuropathy & $7915(3.5)$ & $6093(2.7)$ \\
\hline Secondary osteoporosis & $5284(2.3)$ & $3352(1.5)$ \\
\hline \multicolumn{3}{|c|}{ Drug use within 6 months before index date } \\
\hline Anti-hyperglycemic drugs & $8541(3.7)$ & $8676(3.8)$ \\
\hline Biguanides & $6223(2.7)$ & $6678(2.9)$ \\
\hline Sulfonylurea derivatives & $3900(1.7)$ & $3809(1.7)$ \\
\hline Thiazolidinediones & $262(0.1)$ & $183(0.1)$ \\
\hline Glinides & $87(0.0)$ & $83(0.0)$ \\
\hline GLP-1 RAs & $255(0.1)$ & $220(0.1)$ \\
\hline DPP4-Is & $643(0.3)$ & $707(0.3)$ \\
\hline Insulins & $4900(2.1)$ & $3261(1.4)$ \\
\hline Short acting & $2046(0.9)$ & $1139(0.5)$ \\
\hline Intermediate acting & $2049(0.9)$ & $1411(0.6)$ \\
\hline Long acting & $1444(0.6)$ & $869(0.4)$ \\
\hline Combinations & $1679(0.7)$ & $1200(0.5)$ \\
\hline Statins & $31,874(13.9)$ & $32,064(14.0)$ \\
\hline Antiarrhythmics & $818(0.4)$ & $522(0.2)$ \\
\hline Beta-blockers & $23,281(10.2)$ & $23,592(10.3)$ \\
\hline Thiazide diuretics & $20,425(8.9)$ & $21,547(9.4)$ \\
\hline RAAS inhibitors & $37,555(16.4)$ & $39,379(17.2)$ \\
\hline Calcium channel blockers & $22,942(10.0)$ & $22,816(10.0)$ \\
\hline Loop diuretics & $16,905(7.4)$ & $12,766(5.6)$ \\
\hline Antidepressants & $33,644(14.7)$ & $20,338(8.9)$ \\
\hline Anti-Parkinson drugs & $3174(1.4)$ & $1814(0.8)$ \\
\hline Antipsychotics & $8032(3.5)$ & $4867(2.1)$ \\
\hline Anxiolytics & $14,668(6.4)$ & $10,431(4.6)$ \\
\hline Hypnotics & $19,137(8.4)$ & $14,332(6.3)$ \\
\hline Glucocorticoids & $9390(4.1)$ & $6858(3.0)$ \\
\hline Bisphosphonates & $7371(3.2)$ & $4913(2.1)$ \\
\hline Raloxifene & $214(0.1)$ & $148(0.1)$ \\
\hline
\end{tabular}


Table 1 continued

\begin{tabular}{lcc}
\hline Characteristic & Cases $(n=229,145)$ & Controls $(n=229,145)$ \\
\hline Vitamin D & $211(0.1)$ & $169(0.1)$ \\
Calcium & $1885(0.8)$ & $1375(0.6)$ \\
Strontium ranelate & $194(0.1)$ & $101(0.0)$ \\
PTH & $130(0.1)$ & $70(0.0)$ \\
Calcitonin & $2(0.0)$ & $1(0.0)$ \\
Hormone replacement therapy & $11,912(5.2)$ & $13,955(6.1)$ \\
Beta2-agonists & $10,436(4.6)$ & $8130(3.6)$ \\
Inhaled anticholinergics & $4569(2.0)$ & $3277(1.4)$ \\
Inhaled corticosteroids & $5265(2.3)$ & $4657(2.0)$ \\
\hline
\end{tabular}

Data are number (\%) of patients, unless stated otherwise

$S D$ standard deviation, GLP-1 RA glucagon-like peptide-1 receptor agonist, DPP4-I dipeptidyl peptidase 4 inhibitor, $C O P D$ chronic obstructive pulmonary disease, $R A A S$ renin angiotensin aldosterone system, $P T H$ parathyroid hormone

Table 2 Use of GLP-1 RAs and risk of any fracture

\begin{tabular}{|c|c|c|c|c|}
\hline Exposure & $\begin{array}{l}\text { No. of cases } \\
(N=229,145)^{\mathrm{a}}\end{array}$ & $\begin{array}{l}\text { No. of controls } \\
(N=229,145)^{\mathrm{a}}\end{array}$ & $\begin{array}{l}\text { Crude OR } \\
(95 \% \mathrm{CI})\end{array}$ & $\begin{array}{l}\text { Adjusted OR } \\
(95 \% \mathrm{CI})^{\mathrm{b}}\end{array}$ \\
\hline Never NIAD use & 217,623 & 218,194 & $1.03(0.99-1.06)$ & $1.10(1.06-1.14)^{*}$ \\
\hline Past NIAD use & 3631 & 2815 & $1.33(1.25-1.41)^{*}$ & $1.12(1.05-1.20) *$ \\
\hline Current NIAD use excluding incretin use & 6993 & 7209 & Reference & Reference \\
\hline Past GLP-1 RA use (183-365 days before index date) & 120 & 93 & $1.33(1.01-1.74)^{*}$ & $1.09(0.82-1.46)$ \\
\hline Recent GLP-1 RA use (92-182 days before the index date) & 55 & 56 & $1.01(0.70-1.47)$ & $1.03(0.69-1.53)$ \\
\hline Current GLP-1 RA use (1-91 days before the index date) & 80 & 71 & $1.16(0.84-1.60)$ & $1.16(0.83-1.63)$ \\
\hline \multicolumn{5}{|l|}{ By sex } \\
\hline Males & 38 & 39 & $0.98(0.63-1.54)$ & $0.97(0.60-1.57)$ \\
\hline Females & 42 & 32 & $1.37(0.86-2.18)$ & $1.43(0.87-2.32)$ \\
\hline \multicolumn{5}{|l|}{ By age on index date } \\
\hline$<50$ years & 12 & 10 & $1.28(0.52-3.11)$ & $1.19(0.47-2.98)$ \\
\hline $50-59$ years & 20 & 22 & $0.95(0.51-1.75)$ & $0.99(0.51-1.89)$ \\
\hline $60-69$ years & 33 & 26 & $1.09(0.64-1.86)$ & $1.26(0.71-2.24)$ \\
\hline $70+$ years & 15 & 13 & $1.29(0.60-2.77)$ & $1.04(0.46-2.35)$ \\
\hline
\end{tabular}

Never NIAD use: no NIAD prescription before the index date

Past NIAD use: most recent NIAD prescription over 91 days before index date

Current NIAD use: most recent NIAD prescription within 91 days before index date

$O R$ odds ratio, $C I$ confidence interval, GLP-1 RA glucagon-like peptide-1 receptor agonist, DPP4-I dipeptidyl peptidase 4 inhibitor, NIAD noninsulin anti-diabetic-drugs, COPD chronic obstructive pulmonary disease, $R A A S$ renin-angiotensin-aldosterone system

* Statistically significant, $(P<0.05)$

a The numbers do not add up precisely to the total number of fractures because DPP4-I exposure is not shown

b Adjusted for history of cancer, COPD, fracture, alcoholism, rheumatoid arthritis, secondary osteoporosis, hyperthyroidism, retinopathy, neuropathy, heart failure and use of DPP4-Is, glucocorticoids, statins, anxiolytics, hypnotics, antidepressants, antipsychotics, anti-Parkinson drugs, beta-blockers, thiazide diuretics, RAAS inhibitors, loop diuretics, and antiarrhythmics

\section{Current GLP-1 RA Use Stratified by Cumulative and Average Daily Dose and Fracture Risk}

The risk of any fracture was comparable, and not significantly reduced, among the cumulative and average daily dose groups, Table 4. The adjusted OR for the highest cumulative dose
( $\geq 5.5$ mg exenatide equivalent) was 0.98 (95\% CI 0.50-1.94) and the adjusted OR for the highest average daily dose ( $\geq 22.5 \mathrm{mcg}$ exenatide equivalent per day) was 1.66 (95\% CI 0.86-3.19). When only osteoporotic fractures were considered, there was again no association with a decreased risk of fracture. Adjusted OR for the highest cumulative dose $(\geq 5.5 \mathrm{mg}$ 
Table 3 Use of GLP-1 RAs and fracture risk at different skeletal sites

\begin{tabular}{|c|c|c|c|c|}
\hline Fracture sites & No. of cases ${ }^{\mathrm{a}}$ & No. of controls ${ }^{\mathrm{a}}$ & Crude OR (95\% CI) & Adjusted OR $(95 \% \mathrm{CI})$ \\
\hline Osteoporotic fracture & 96,774 & 96,774 & - & - \\
\hline Never NIAD use & 90,297 & 90,677 & $1.02(0.97-1.07)$ & $1.07^{\mathrm{b}}(1.02-1.13)^{*}$ \\
\hline Current NIAD use excl. incretin use & 3957 & 4058 & Reference & Reference \\
\hline Past GLP-1 RA use ( $>182$ days before the index date) & 47 & 41 & $1.17(0.77-1.78)$ & $0.94^{\mathrm{b}}(0.60-1.48)$ \\
\hline Recent GLP-1 RA use (92-182 before the index date) & 23 & 24 & $0.98(0.55-1.73)$ & $0.97^{\mathrm{b}}(0.52-1.79)$ \\
\hline Current GLP-1 RA use (0-91 days before the index date) & 23 & 31 & $0.76(0.44-1.30)$ & $0.78^{\mathrm{b}}(0.44-1.39)$ \\
\hline Hip & 24,328 & 24,328 & - & - \\
\hline Never NIAD use & 21,923 & 22,273 & $0.94(0.87-1.01)$ & $1.00^{\mathrm{c}}(0.92-1.09)$ \\
\hline Current NIAD use excl. incretin use & 1454 & 1388 & Reference & Reference \\
\hline Past GLP-1 RA use ( $>182$ days before the index date) & 9 & 4 & $2.11(0.65-6.86)$ & $1.74^{\mathrm{c}}(0.47-6.49)$ \\
\hline Recent GLP-1 RA use (92-182 before the index date) & 5 & 4 & $1.18(0.32-4.40)$ & $1.34^{\mathrm{c}}(0.26-6.93)$ \\
\hline Current GLP-1 RA use (0-91 days before the index date) & 4 & 4 & $0.99(0.25-3.97)$ & $0.77^{\mathrm{c}}(0.16-3.73)$ \\
\hline Radius/ulna & 47,905 & 47,905 & - & - \\
\hline Never NIAD use & 45,848 & 45,423 & $1.29(1.19-1.39)^{*}$ & $1.29^{\mathrm{d}}(1.19-1.40)^{*}$ \\
\hline Current NIAD use excl. incretin use & 1273 & 1617 & Reference & Reference \\
\hline Past GLP-1 RA use ( $>182$ days before the index date) & 19 & 21 & $1.16(0.62-2.16)$ & $0.93^{\mathrm{d}}(0.48-1.80)$ \\
\hline Recent GLP-1 RA use (92-182 before the index date) & 11 & 12 & $1.18(0.52-2.68)$ & $1.32^{\mathrm{d}}(0.55-3.17)$ \\
\hline Current GLP-1 RA use (0-91 days before the index date) & 11 & 20 & $0.70(0.34-1.47)$ & $0.93^{\mathrm{d}}(0.48-1.80)$ \\
\hline Vertebral & 9004 & 9004 & - & - \\
\hline Never NIAD use & 8396 & 8450 & $1.01(0.87-1.17)$ & $1.11^{\mathrm{e}}(0.93-1.32)$ \\
\hline Current NIAD use excl. incretin use & 364 & 369 & Reference & Reference \\
\hline Past GLP-1 RA use (>182 days before the index date) & 4 & 5 & $0.80(0.21-3.02)$ & $0.97^{\mathrm{e}}(0.22-4.32)$ \\
\hline Recent GLP-1 RA use (92-182 before the index date) & 2 & 4 & $0.50(0.09-2.75)$ & $0.30^{\mathrm{e}}(0.05-1.84)$ \\
\hline Current GLP-1 RA use (0-91 days before the index date) & 2 & 2 & $1.00(0.14-7.15)$ & $2.02^{\mathrm{e}}(0.28-14.75)$ \\
\hline
\end{tabular}

Never NIAD use: no NIAD prescription before the index date

Current NIAD use: most recent NIAD prescription within 91 days before index date

$O R$ odds ratio, $C I$ confidence interval, $G L P-1 R A$ glucagon-like peptide-1 receptor agonist, DPP4-I dipeptidyl peptidase 4 inhibitor, NIAD noninsulin anti-diabetic-drugs, $C O P D$ chronic obstructive pulmonary disease, $R A A S$ renin-angiotensin-aldosterone system

* Statistically significant, $(P<0.05)$

a The numbers do not add up precisely to the total number of fractures because past NIAD use and DPP4-I exposure are not shown

b Adjusted for (f) and history of retinopathy, heart failure, COPD and use of glucocorticoids, statins, anxiolytics, antipsychotics, beta-blockers, thiazide diuretics, RAAS inhibitors, and antiarrhythmics

c Adjusted for (f) and history of retinopathy, heart failure, COPD and use of statins, anxiolytics, RAAS inhibitors, antiarrhythmics, glucocorticoids, and antipsychotics

d Adjusted for (f) and use of beta-blockers, thiazide diuretics, RAAS inhibitors, and calcium channel inhibitors

e Adjusted for (f) and history of COPD and use of glucocorticoids, anxiolytics, RAAS inhibitors and antipsychotics

${ }^{\mathrm{f}}$ History of cancer, alcoholism, fracture, rheumatoid arthritis and secondary osteoporosis and use of DPP4-Is, antidepressants, anti-Parkinson drugs, loop diuretics, and hypnotics

exenatide equivalent) was 0.93 (95\% CI 0.30-2.92) and the adjusted OR for the highest average daily dose $(\geq 22 \mathrm{mcg}$ exenatide equivalent per day) was 1.40 (95\% CI 0.47-4.13).

\section{Sensitivity Analysis}

Adjusting the main analysis for current metformin use did not substantially change our results. The adjusted OR for current GLP-1 RA use was 1.17 (95 \% CI 0.83-1.64), for recent GLP-1 RA 1.03 (95\% CI 0.70-1.54), and for past GLP-1 RA use 1.09 (95 \% CI 0.82-1.46).

As a second sensitivity analysis, we additionally adjusted the main analysis for insulin use and this did not alter the results. The adjusted OR for current GLP-1 RA use was $1.13(0.80-1.58)$, for recent use $1.00(0.67-1.49)$, and for past use $1.05(0.79-1.41)$. 
Table 4 Use of GLP-1 RAs and risk of any fracture stratified by cumulative and average daily dose

\begin{tabular}{|c|c|c|c|c|c|c|}
\hline & \multicolumn{3}{|l|}{ Any fracture } & \multicolumn{3}{|c|}{ Osteoporotic fracture } \\
\hline & $\begin{array}{l}\text { No. of cases } \\
(N=229,145)^{\mathrm{a}}\end{array}$ & $\begin{array}{l}\text { No. of controls } \\
(N=229,145)^{\mathrm{a}}\end{array}$ & $\begin{array}{l}\text { Adjusted OR } \\
(95 \% \mathrm{CI})^{\mathrm{b}}\end{array}$ & $\begin{array}{l}\text { No. of cases } \\
(N=96,774)^{\mathrm{a}}\end{array}$ & $\begin{array}{l}\text { No. of controls } \\
(N=96,774)^{\mathrm{a}}\end{array}$ & $\begin{array}{l}\text { Adjusted OR } \\
(95 \% \mathrm{CI})^{\mathrm{c}}\end{array}$ \\
\hline Current NIAD use excl. incretins & 6993 & 7209 & Reference & 3957 & 4058 & Reference \\
\hline Current GLP-1 RA use & 80 & 71 & $1.16(0.83-1.63)$ & 23 & 31 & $0.78(0.44-1.39)$ \\
\hline \multicolumn{7}{|l|}{ By cumulative exposure $^{\mathrm{d}}$} \\
\hline $0-1.4 \mathrm{mg}$ & 36 & 34 & $1.05(0.63-1.73)$ & 10 & 17 & $0.64(0.28-1.47)$ \\
\hline $1.4-2.7 \mathrm{mg}$ & 14 & 11 & $1.60(0.69-3.69)$ & 3 & 5 & $0.57(0.12-2.74)$ \\
\hline $2.7-5.5 \mathrm{mg}$ & 12 & 8 & $1.55(0.60-3.95)$ & 4 & 2 & $2.05(0.32-13.30)$ \\
\hline$\geq 5.5 \mathrm{mg}$ & 18 & 18 & $0.98(0.50-1.94)$ & 6 & 7 & $0.93(0.30-2.92)$ \\
\hline \multicolumn{7}{|l|}{ By average daily dose ${ }^{\mathrm{d}}$} \\
\hline$<15 \mathrm{mcg} /$ day & 16 & 19 & $0.91(0.47-1.84)$ & 3 & 9 & $0.35(0.09-1.39)$ \\
\hline $15-22.49 \mathrm{mcg} / \mathrm{day}$ & 38 & 36 & $1.07(0.66-1.73)$ & 12 & 15 & $0.78(0.35-1.75)$ \\
\hline$\geq 22.5 \mathrm{mcg} /$ day & 26 & 16 & $1.66(0.86-3.19)$ & 8 & 7 & $1.40(0.47-4.13)$ \\
\hline Recent GLP-1 RA use & 55 & 56 & $1.03(0.69-1.53)$ & 23 & 24 & $0.97(0.52-1.79)$ \\
\hline Past GLP-1 RA use & 120 & 93 & $1.09(0.82-1.46)$ & 47 & 41 & $0.94(0.60-1.48)$ \\
\hline
\end{tabular}

$O R$ odds ratio, $C I$ confidence interval, GLP-1 RA glucagon-like peptide-1 receptor agonist, DPP4-I dipeptidyl peptidase 4 inhibitor, NIAD noninsulin anti-diabetic-drugs, $C O P D$ chronic obstructive pulmonary disease, $R A A S$ renin-angiotensin-aldosterone system

* Statistically significant, $(P<0.05)$

${ }^{\text {a }}$ The numbers do not add up precisely to the total number of fractures because never NIAD, past NIAD and DPP4-I use are not shown

b Adjusted for history of cancer, alcoholism, COPD, fracture, rheumatoid arthritis, hyperthyroidism, secondary osteoporosis, retinopathy, neuropathy and heart failure and use of DPP4-Is, glucocorticoids, statins, antidepressants, anxiolytics, hypnotics, antipsychotics, anti-Parkinson drugs, beta-blockers, thiazide diuretics, RAAS inhibitors, loop diuretics, antiarrhythmics

c Adjusted for (a), but not for hyperthyroidism and neuropathy

${ }^{\mathrm{d}}$ In exenatide equivalents

\section{Discussion}

The present study showed that current GLP-1 RA use was not associated with a decreased risk of any fracture, as compared to the use of other anti-hyperglycemic drugs, and current GLP-1 RA use was not associated with a reduced risk of other fracture types. Moreover, stratification of current GLP-1 RA by cumulative or average daily dose was not associated with a decreased risk of fracture.

The results of the present study are in line with the results of a meta-analysis of clinical trials on the effect of GLP-1 RAs on fracture risk, which showed that fracture risk was not significantly reduced with use of GLP-1 RA $[11,23]$. Our results are also in keeping with a large clinical trial $(n=16,492)$ on the effect of a DPP4-I, saxagliptin, which showed no difference in risk of fracture with the use of DPP4-I use and placebo [24]. Additionally, the present results are also supported by the results of a cohort study which was not able to show a decreased risk of fracture with the use of GLP-RA as compared to the use of other anti-hyperglycemic drugs [12] and a cohort study that compared the use of DPP4-I to the use of other antihyperglycemic drugs [25]. The pathway by which DPP4-Is might affect bone metabolism may be the same as that of GLP-1 RA because DPP4-Is inhibits the degradation of GLP-1 [2]. Moreover, our results are indirectly supported by a clinical trial on the effect of a GLP-1 RA, exenatide, on markers of bone metabolism [26], which reported that bone markers were unaffected after 44 weeks of exenatide treatment.

We acknowledge that our findings are in contrast to those of a meta-analysis of randomized clinical trials $(n=22,055)$ that showed a significant $40 \%$ reduction of fracture risk with the use of DPP4-Is [27] as compared to active treatment or placebo. However, studies that were included in this meta-analysis did not routinely collect fractures as an outcome of interest, the number of fractures was low, and different comparator groups had been used [27]. The results from this meta-analysis should likely be interpreted with caution as the low number of events in a meta-analysis of adverse events can give biased estimates $[28,29]$.

Results of in vitro studies have suggested that the use of GLP-1 RAs might have a beneficial effect on bone metabolism [7-9], yet different mechanisms of effect have been hypothesized. In vitro studies have shown that GLP-1 
receptors are present on bone marrow stromal cells [30], immature osteoblast [31] and osteocytes [10], and binding of GLP-1 to its receptor on bone cells leads to increased osteoblast activity [9], and decreased osteoclast activity [10]. This could then lead to increased bone formation and a reduced fracture risk. However, more research is needed to assess whether GLP-1 RAs are also able to bind to GLP1 receptors on human bone cells and whether this could ultimately result in a reduced risk of fracture.

An unexpected finding was that never and past NIAD use showed an increased fracture risk as compared to the current use of other anti-hyperglycemic drugs. This may have been the result of residual confounding by high body mass index (BMI), which has been shown to decrease fracture risk [32]. T2DM has been associated with an increased BMI and therefore it might be that the increased risk of never NIAD use is a result of a higher BMI in the current NIAD use group which was used as a reference group. The past NIAD use group included patients with T2DM who use insulin only. Insulin use has been associated with a twofold increased risk of fracture as compared to patients with T2DM who use NIADs [2].

There are several strengths to the current study. First the use of a nation-wide population register permitted the examination of a large number of cases and controls. The data used were also collected longitudinally, and for prescriptions this permitted us to calculate a reliable cumulative and average daily dose. Second, the data used to identify fractures have been validated [13]. Third, we were able to adjust our analyses for many potential confounders. When interpreting our results, we are mindful of a couple of limitations. We were not able to adjust for potential confounders such as BMI, hemoglobin A1c (HbA1c), smoking, amount of exercise, and serum vitamin D levels. A high BMI has been associated with a reduced risk of fracture [2] and therefore a decreased risk of fracture with GLP-1 RA use could have represented the association between high BMI and fracture risk. Nevertheless, we did not show a reduced fracture risk with GLP-1 RA use. Smoking is a risk factor of fracture [33], but there is no evidence that GLP-1 RA users have different smoking behaviors than the patients treated with other anti-hyperglycemic drugs. Exercise has been associated with a decreased risk of fracture [34]; GLP-1 RA users might perform less exercise which could result in an overestimation of the effect. Lower levels of serum vitamin D have been associated with more severe T2DM complications and increased fracture risk [35, 36]. Not adjusting for serum vitamin D levels might lead to an overestimation of the effect when GLP-1 RA users have lower serum vitamin D levels as compared to the users of other anti-hyperglycemic drugs. Thus, while we were able to identify a large number of confounders due to the completeness of the registry data, it is acknowledged that some residual confounding might still be present. In addition, we tried to capture severity of diabetes by correcting our analyses for known complications of diabetes, such as neuropathy and retinopathy. Moreover, we used current NIAD use (excluding incretin use) as a reference group, because diabetes itself might act as a confounder. Current NIAD use included use of TZDs or insulins, which have been shown to increase fracture risk $[3,4]$. The result of this bias in the reference category may be an observed artificial inverse association between GLP-1 RAs use and risk of fracture which could have falsely supported our hypothesis. However, we did not observe an inverse association between GLP-1 RA use and risk of fracture. Current NIAD use also included metformin use which has been associated with a reduced fracture risk and therefore it might mask a decreased association between GLP-1 RA use and fracture risk. Nevertheless, statistical adjustment of the main analysis for current metformin use did not alter the results.

Although the total number of fractures was high, the number of some fracture types (i.e., hip and radius/ulna) was not high enough to stratify current GLP-1 RA use by cumulative and average dose, and to keep adequate statistical power. In the analyses, with hip and vertebral fracture as outcome, the number of GLP-1 RA users was quite small, therefore these results should be interpreted with caution. The average duration of actual GLP-1 RA use (36 weeks) in the present study was rather short, which might be the reason that we were unable to observe an association between GLP-1 RA use and fracture risk. However, after stratification of current GLP-1 RA use by cumulative dose, risk of fracture was not decreased in the group with patients who had used on average 15 microgram exenatide equivalent per day for at least 1 year (cumulative dose: $\geq 5.5 \mathrm{mg}$ exenatide equivalent).

In conclusion, we showed in a population-based casecontrol study that the use of GLP-1 RAs (current, recent or past) is not associated with fracture risk as compared to the use of other anti-hyperglycemic drugs. In addition, current GLP-1 RA use stratified by cumulative and average daily dose was not associated with a decreased fracture risk. More research is needed, and in particular future studies should focus on the effect of long-term use of GLP-1 RAs on fracture risk.

Acknowledgments Andrea M. Burden is supported by a Canadian Institutes of Health Research (CIHR) Post Doctoral Fellowship. Statistics Denmark is acknowledged for the help with the acquisition of the data.

\section{Compliance with Ethical Standards}

Conflict of interest Johanna H. M. Driessen, Frank de Vries and Andrea M. Burden declare that they are employed by the Division of 
Pharmacoepidemiology and Clinical Pharmacology at Utrecht Institute for Pharmaceutical Sciences, which has received unrestricted funding from the Netherlands Organisation for Health Research and Development (ZonMW), the Dutch Health Care Insurance Board (CVZ), the Royal Dutch Pharmacists Association (KNMP), the private-public-funded Top Institute Pharma (www.tipharma.nl), including co-funding from universities, government, and industry, the EU Innovative Medicines Initiative (IMI), the EU 7th Framework Program (FP7), the Dutch Ministry of Health and industry (including GlaxoSmithKline, Pfizer, and others).

Human and Animal Rights and Informed Consent This article does not contain any studies with human or animal subjects performed by any of the authors. Informed consent was obtained from all individual participants included in the study.

Open Access This article is distributed under the terms of the Creative Commons Attribution 4.0 International License (http://crea tivecommons.org/licenses/by/4.0/), which permits unrestricted use, distribution, and reproduction in any medium, provided you give appropriate credit to the original author(s) and the source, provide a link to the Creative Commons license, and indicate if changes were made.

\section{References}

1. Vestergaard P (2007) Discrepancies in bone mineral density and fracture risk in patients with type 1 and type 2 diabetes-a metaanalysis. Osteoporos Int 18(4):427-444

2. Montagnani A, Gonnelli S (2013) Antidiabetic therapy effects on bone metabolism and fracture risk. Diabetes Obes Metab 15(9):784-791

3. Bazelier MT, Gallagher AM, van Staa TP, Cooper C, Leufkens HG, Vestergaard P et al (2012) Use of thiazolidinediones and risk of osteoporotic fracture: disease or drugs? Pharmacoepidemiol Drug Saf 21(5):507-514

4. Betteridge DJ (2011) Thiazolidinediones and fracture risk in patients with type 2 diabetes. Diabet Med 28(7):759-771

5. Schwartz AV, Vittinghoff E, Bauer DC, Hillier TA, Strotmeyer ES, Ensrud KE et al (2011) Association of BMD and FRAX score with risk of fracture in older adults with type 2 diabetes. JAMA 305(21):2184-2192

6. Vestergaard P, Rejnmark L, Mosekilde L (2005) Relative fracture risk in patients with diabetes mellitus, and the impact of insulin and oral antidiabetic medication on relative fracture risk. Diabetologia 48(7):1292-1299

7. Nuche-Berenguer B, Moreno P, Esbrit P, Dapía S, Caeiro JR, Cancelas J et al (2009) Effect of GLP-1 treatment on bone turnover in normal, type 2 diabetic, and insulin-resistant states. Calcif Tissue Int 84:453-461

8. Nuche-Berenguer B, Moreno P, Portal-Nuñez S, Dapía S, Esbrit P, Villanueva-Peñacarrillo ML (2010) Exendin-4 exerts osteogenic actions in insulin-resistant and type 2 diabetic states. Regul Pept 159(1-3):61-66

9. Moreno P, Nuche-Berenguer B, Gutiérrez-Rojas I, Acitores A, Sancho V, Valverde I et al (2012) Normalizing action of exendin4 and GLP-1 in the glucose metabolism of extrapancreatic tissues in insulin-resistant and type 2 diabetic states. J Mol Endocrinol 48:37-47

10. Kim JY, Lee SK, Jo KJ, Song DY, Lim DM, Park KY et al (2013) Exendin-4 increases bone mineral density in type 2 diabetic OLETF rats potentially through the down-regulation of SOST/ sclerostin in osteocytes. Life Sci 92(10):533-540
11. Mabilleau G, Mieczkowska A, Chappard D (2014) Use of glucagon-like peptide-1 receptor agonists and bone fractures: a meta-analysis of randomized clinical trials. J Diabetes 6(3):260-266

12. Driessen JH, Henry RM, van Onzenoort HA, Lalmohamed A, Burden AM, Prieto-Alhambra D et al (2015) Bone fracture risk is not associated with the use of glucagon-like peptide-1 receptor agonists: a population-based cohort analysis. Calcif Tissue Int:1-9 [Epub ahead of print]

13. Vestergaard P, Mosekilde L (2002) Fracture risk in patients with celiac disease, Crohn's disease, and ulcerative colitis: a nationwide follow-up study of 16,416 patients in Denmark. Am J Epidemiol 156(1):1-10

14. Frank L (2000) Epidemiology. When an entire country is a cohort. Science 287:2398-2399

15. Andersen T, Madsen M, Jørgensen J, Mellemkjær L, Olsen J (1999) The Danish National Hospital Register. Dan Med Bull 46:263-268

16. Wacholder S, McLaughlin J, Silverman D, Mandel J (1992) Selection of controls in case-control studies. I. Principles. Am J Epidemiol 135:1019-1028

17. FRAX. WHO Fracture Risk Assessment Tool 2001. shef.ac.uk. www.shef.ac.uk/FRAX/index.aspx

18. Anonymous (2002) ATC classification index with DDDs 2002. Nydalen, WHO Collaborating Centre for Drug Statistics Methodology, Norwegian Institute of Public Health

19. Klop C, de Vries F, Vinks T, Kooij MJ, van Staa TP, Bijlsma JW et al (2014) Increase in prophylaxis of glucocorticoid-induced osteoporosis by pharmacist feedback: a randomised controlled trial. Osteoporos Int 25(1):385-392

20. Prieto-Alhambra D, Petri H, Goldenberg JS, Khong TP, Klungel $\mathrm{OH}$, Robinson NJ et al (2014) Excess risk of hip fractures attributable to the use of antidepressants in five European countries and the USA. Osteoporos Int 25(3):847-855

21. Khong TP, de Vries F, Goldenberg JS, Klungel OH, Robinson NJ, Ibáñez L et al (2012) Potential impact of benzodiazepine use on the rate of hip fractures in five large European countries and the United States. Calcif Tissue Int 91(1):24-31

22. Pouwels S, Bazelier MT, de Boer A, Weber WE, Neef C, Cooper C et al (2013) Risk of fracture in patients with Parkinson's disease. Osteoporos Int 24(8):2283-2290

23. Su B, Sheng H, Zhang M, Bu L, Yang P, Li L et al (2015) Risk of bone fractures associated with glucagon-like peptide-1 receptor agonists' treatment: a meta-analysis of randomized controlled trials. Endocrine 48(1):107-115

24. Scirica BM, Bhatt DL, Braunwald E, Steg PG, Davidson J, Hirshberg B et al (2013) Saxagliptin and cardiovascular outcomes in patients with type 2 diabetes mellitus. $\mathrm{N}$ Engl J Med 369(14):1317-1326

25. Driessen JH, van Onzenoort HA, Henry RM, Lalmohamed A, van den Bergh JP, Neef C et al (2014) Use of dipeptidyl peptidase-4 inhibitors for type 2 diabetes mellitus and risk of fracture. Bone 68:124-130

26. Bunck MC, Eliasson B, Corner A, Heine RJ, Shaginian RM, Taskinen MR et al (2011) Exenatide treatment did not affect bone mineral density despite body weight reduction in patients with type 2 diabetes. Diabetes Obes Metab 13:374-377

27. Monami M, Dicembrini I, Antenore A, Mannucci E (2011) Dipeptidyl peptidase-4 inhibitors and bone fractures: a meta-analysis of randomized clinical trials. Diabetes Care 34(11):2474-2476

28. Lane PW (2013) Meta-analysis of incidence of rare events. Methods Med Res 22(2):117-132

29. Bhaumik DK, Amatya A, Normand SL, Greenhouse J, Kaizar E, Neelon B et al (2012) Meta-analysis of rare binary adverse event data. J Am Stat Assoc 107(498):555-567 
30. Sanz C, Vázquez P, Blázquez C, Barrio PA, del Alvarez MM, Blázquez E (2010) Signaling and biological effects of glucagonlike peptide 1 on the differentiation of mesenchymal stem cells from human bone marrow. Am J Physiol Endocrinol Metab 298(3):E634-E643

31. Phillips LK, Prins JB (2011) Update on incretin hormones. Ann N Y Acad Sci 1243:E55-E74

32. Cummings SR, Nevitt MC, Browner WS, Stone K, Fox KM, Ensrud KE et al (1995) Risk factors for hip fracture in white women. Study of Osteoporotic Fractures Research Group. N Engl J Med 332(12):767-773

33. Kanis JA, Johnell O, Oden A, Johansson H, De Laet C, Eisman JA et al (2005) Smoking and fracture risk: a meta-analysis. Osteoporos Int 16(2):155-162
34. Kemmler W, Häberle L, von Stengel S (2013) Effects of exercise on fracture reduction in older adults: a systematic review and meta-analysis. Osteoporos Int 24(7):937-950

35. Zoppini G, Galletti A, Targher G, Brangani C, Pichiri I, Trombetta $\mathrm{M}$ et al (2015) Lower levels of 25-hydroxyvitamin D3 are associated with a higher prevalence of microvascular complications in patients with type 2 diabetes. BMJ Open Diabetes Res Care 3(1): 000058

36. Bischoff-Ferrari HA, Willett WC, Wong JB, Stuck AE, Staehelin HB, Orav EJ et al (2009) Prevention of nonvertebral fractures with oral vitamin $\mathrm{D}$ and dose dependency: a meta-analysis of randomized controlled trials. Arch Intern Med 169(6):551-561 